\title{
SIMULACIÓN Y DISEÑO DE UNA MESA DE BIPEDESTACIÓN PARA PA- CIENTES CON TRAUMA RAQUIMEDULAR DE NIVEL BAJO O LESIÓN EN CAUDA EQUINA
}

\author{
SIMULATION AND DESIGN OF A STANDING TABLE FOR PATIENTS WITH LOW-LEVEL SPI- \\ NAL CORD INJURY OR CAUDA EQUINA INJURY
}

Gabriel Felipe González Orozcoํ, Gonzalo Guillermo Moreno Contreras², Emily Gómez Lopez³.

\footnotetext{
${ }^{1}$ Universidad de Pamplona, Centro de Fisioterapia Cyriax, Pasto - Colombia, gfgo033@hotmail.com.

${ }^{2}$ Universidad de Pamplona, Departamento de Ingeniería Mecánica, gmoren@hotmail.com

${ }^{3}$ Fisioterapia- Universidad Mariana, Centro de Fisioterapia Cyriax- Pasto - Colombia, emilygomezlopez@yahoo.es.
}

\begin{abstract}
RESUMEN
El presente proyecto muestra toda la metodología utilizada para el diseño de un equipo de terapia para la rehabilitación física de pacientes que padezcan trauma raquimedular de nivel bajo, lesión en cauda equina o hernia discal, la cual busca brindar un tratamiento fisioterapéutico más eficiente que los ofrecidos en la actualidad, en este documento se presenta todo el proceso de diseño del equipo siguiendo los estándares previstos para la fabricación de estos equipos electromecánicos.
\end{abstract}

Palabras claves: Mesa de bipedestación, columna vertebral, hernia discal, lesión en cauda equina, Centro de Fisioterapia Cyriax.

\begin{abstract}
This project shows all the methodology used to design equipment for the physical therapy of patients suffering from low-level spinal cord injury, cauda equine injury or herniated disc, which seeks to provide a more efficient physiotherapy that currently offered, in this document contains all the equipment design process following the standards established for the manufacture of these electro mechanical equipment.
\end{abstract}

Keywords words: Table of standing, spine, herniated disc, cauda equine injury, Cyriax Physiotherapy Centre. 


\section{INTRODUCCIÓN}

El vertiginoso incremento de automatismos, obliga al mundo a estar a la vanguardia en la búsqueda de nuevas aplicaciones tecnológicas en los cuales se optimicen los conceptos de vida con calidad, razón por la cual en este caso específico se realiza el diseño de un equipo de mecanoterapia con el fin de aportar más tecnología en el área de la salud, concretamente a la profesión de Fisioterapia (1).

Esta profesión en particular cada día está buscando nuevas tendencias tecnológicas que no solamente sean eficientes para el tratamiento de los cuadros clínicos, sino que minimicen el gasto del fisioterapeuta al realizar los procedimientos manuales de rigor.

La historia indica que entre 1815 y 1825 aparecieron en Inglaterra unas camas de hospital, las cuales podían subir o bajar sus partes accionando unas manivelas; Posteriormente el Dr. Willis D. Gatch (1878-1954), Presidente del Departamento de Cirugía en la Escuela de Medicina de la Universidad de Indiana, inventó la cama "Gatch" ó cama del hospital ajustable, esta cama permite la elevación de la cabeza o los pies independientemente (en realidad, las rodillas) de un paciente debido a la mecánica de sus secciones divididas, esta cuenta con tres sub-secciones divididas con tres elevaciones independientes (2).

La cama Gatch ha servido durante mucho tiempo como base para la creación de equipos cuyo objetivo principal es el tratamiento y mejoramiento de la postura de las personas.

El diseño de la mesa de bipedestación esta encaminado a fusionar dos equipos en uno, el primero es una mesa para el tratamiento de tracción lumbar gravitacional y el segundo es una mesa para el tratamiento de patologías osteomusculares, lo cual permitirá un significativo ahorro en espacio y dinero.

\section{MATERIALES Y MÉTODOS}

\section{A. Selección}

Es importante resaltar que para el proceso de selección y diseño del modelo a seguir se hizo un estudio teniendo en cuenta el tipo de lesiones a tratar, esto permitió seleccionar algunos equipos médicos existente en el mercado y tomar lo mejor de ellos para ser aplicado en el equipo a desarrollar.
Mesa de bipedestación marca Muhamed: Este equipo permite únicamente bipedestar al paciente, lo cual limita su accionar a tratamientos de tracción gravitacional, en la figura 1 se puede observar el equipo descrito.

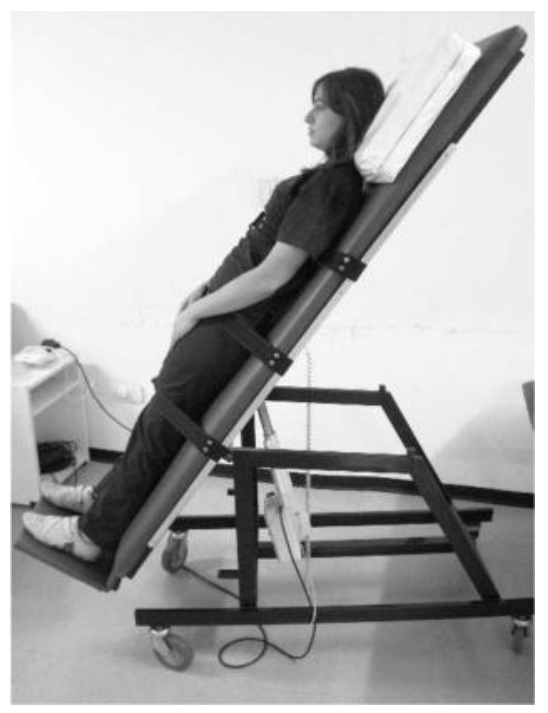

Figura 1. Mesa Muhamed.

Mesa de bipedestación marca Triton: Este equipo permite únicamente la inclinación gradual de los miembros inferiores de los pacientes a tratar, en la figura 2 se puede observar el equipo descrito.

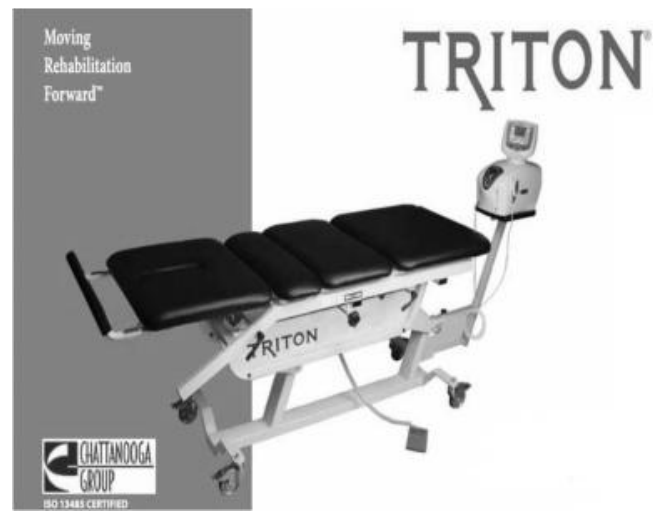

Figura 2. Mesa Triton.

\section{B. Dimensionamiento}

Partiendo del hecho de que una de las grandes metas de la rehabilitación física es que el paciente logre recobrar su movilidad, se tiene como fin principal implementar un equipo que cumpla con las especificaciones dadas por la experiencia del fisioterapeuta y las sugerencias de los pacientes, en cuanto a medidas y comodidad. Por lo anterior, se 
realizó una muestra con 40 pacientes de ambos sexos del Centro de Fisioterapia Cyriax, a los cuales se les realiza tratamiento de rehabilitación física de trauma raquimedular de nivel bajo o con lesión en cauda equina.

\section{RESULTADOS}

De este estudio se pueden destacar los siguientes resultados objetos del diseño a implementar:

Las medidas efectivas (medidas que puede ocupar el paciente): Altura - Largo: $197 \mathrm{~cm}$. (Largo de la mesa.)

Ancho de la espalda: $83 \mathrm{~cm}$. (Ancho de la mesa.), Peso: $90 \mathrm{~kg}$

Con el estudio de selección y dimensionamiento realizado y teniendo en cuenta las recomendaciones hechas por los posibles pacientes, se realizó la propuesta que se indica en la figura 3 , esta mesa permite la bipedestación del paciente y las inclinaciones graduales tanto de la parte superior del cuerpo del paciente como de sus miembros inferiores.

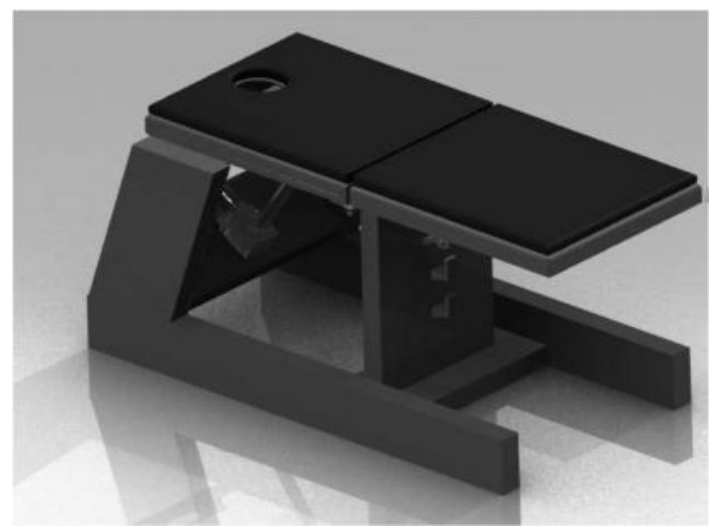

Figura 3. Mesa Bipedestación - Cyriax

Inicialmente se verificó mediante el software de simulación SolidWork ${ }^{\circledR}$ que la Mesa de Bipedestación - Cyriax cumpliera con las funciones dispuestas para el tratamiento de pacientes que padezcan trauma raquimedular de nivel bajo o lesión en cauda equina.

En las figuras 4 y 6 se puede observar la inclinación gradual de la parte inferior de la mesa, esta inclina- ción se hace manualmente.

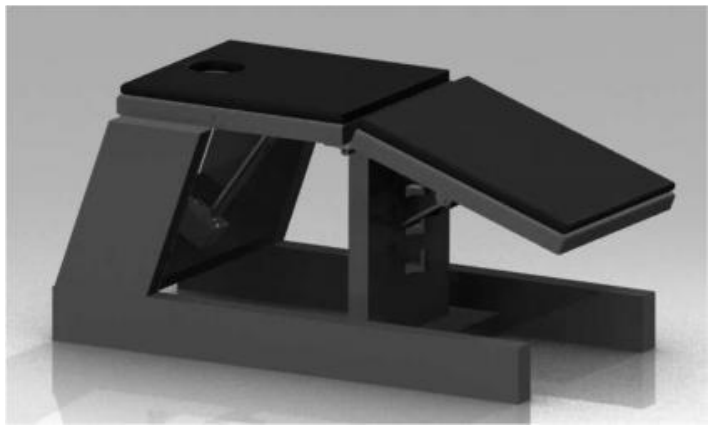

Figura 4. Mesa Bipedestación - Cyriax Postura 1.

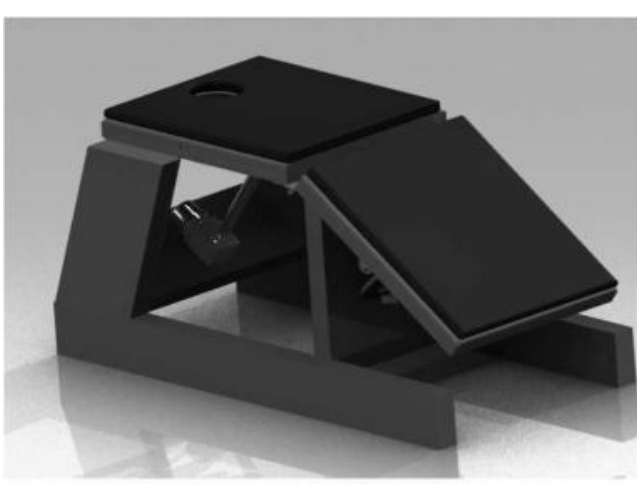

Figura 5. Mesa Bipedestación - Cyriax Postura 2

En las figuras 6 - 9 se puede observar la inclinación gradual y total de la mesa, la inclinación de la parte superior de la mesa es realizada mediante el accionamiento del mecanismo de un tornillo sinfín.

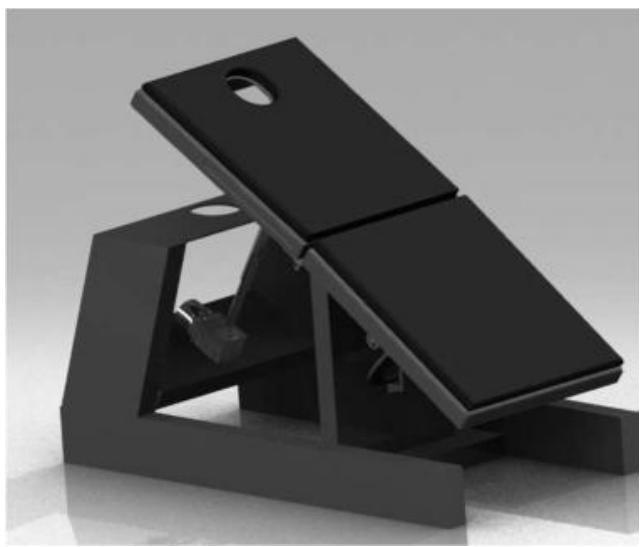

Figura 6. Mesa Bipedestación - Cyriax Postura 3 


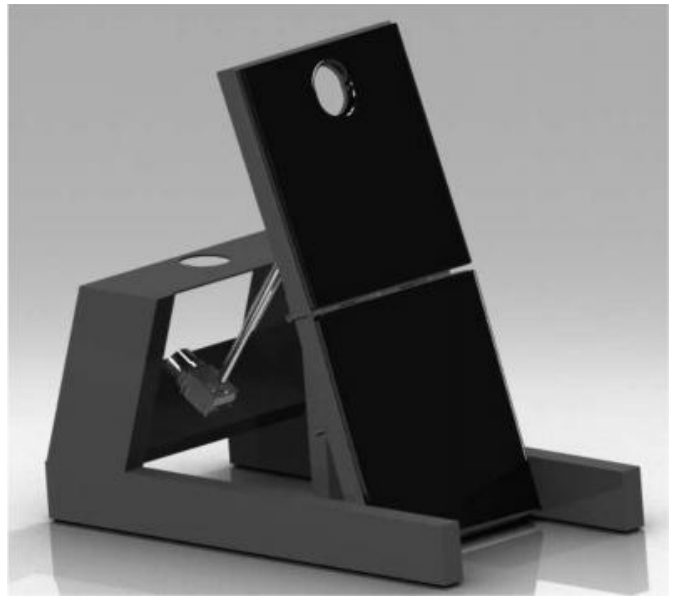

Figura 7. Mesa Bipedestación - Cyriax Postura 4

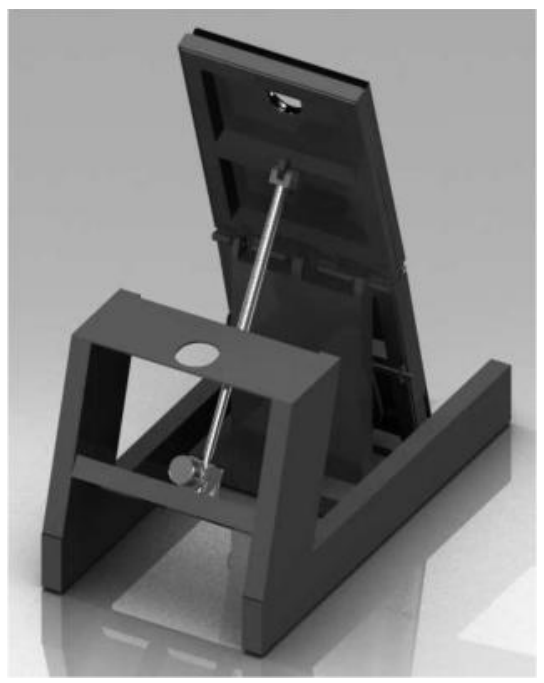

Figura 8. Mesa Bipedestación - Cyriax Postura 4

Una vez verificada la versatilidad del equipo se procedió a realizar el diseño y simulación de los componentes sometidos a cargas máximas.

La mesa de bipedestación esta dividida en dos partes que soportaran el peso del paciente, las patas y la parte superior ó camilla. La camilla a su vez se divide en dos partes una que soporta la parte superior del cuerpo humano (de la cintura hacia arriba) que es accionada por un tornillo sinfín y otra parte donde descansan las piernas, la cual se acciona manualmente.

Como ya se indico anteriormente el peso aproxi- mado de los pacientes a tratar es de $90 \mathrm{~kg}$, realizando una comparación antropométrica del cuerpo humano se estima que de la cintura para arriba el peso del cuerpo humano es del orden del 60 al 70 $\%$ y de la cintura hacia abajo (miembros inferiores) el peso es del orden del 30 al 40\% $(3,4)$, este dato es de suma importancia ya que nos indica un valor real de las cargas que se deben aplicar al momento de hacer los cálculos estructurales y las simulaciones de los diferentes componentes de la cama.

Patas: La estructura interna esta hecha en tubo cuadrado estructural de acero inoxidable $\left(2 \frac{1}{2}\right.$ "X $21 / 2$ "x 1/8") y recubierta con lámina del mismo material.

Para los procesos de calculo, diseño y simulación de las patas se determino que el mayor esfuerzo ocurre cuando el tornillo sinfín acciona gradualmente la parte de la camilla que soporta la parte superior del cuerpo humano, por consiguiente se tomo como parte fija el apoyo en el piso y se aplico una carga de $750 \mathrm{~N}(90 \mathrm{~kg}$ x $70 \%$ x $9.81 \mathrm{~m} / \mathrm{seg} 2$ x $1.2=741.636 \mathrm{~N}$ ) en el apoyo del motor, para lo cual se utilizo un factor de seguridad de 1.2.

En las figuras 9 - 12 se puede observar el proceso de diseño y simulación de las patas, la figura 9 muestra los puntos de aplicación de apoyos y cargas.

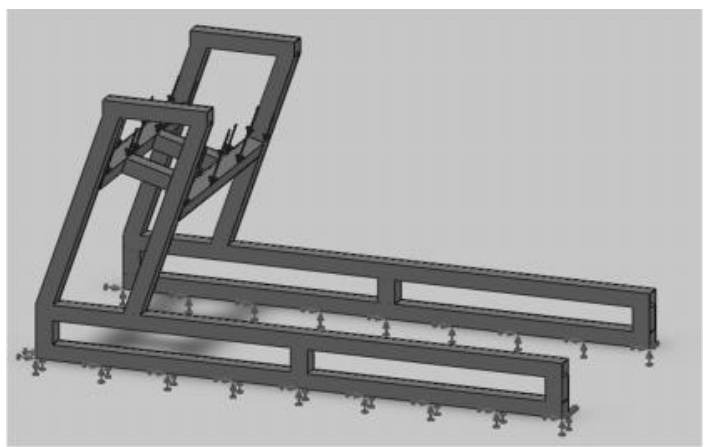

Figura 9. Cargas Estructura de las Patas SolidWorks ${ }^{\circledR}$

La figura 10 indica las deformaciones máximas que se pueden llegar a presentar en la estructura, en este caso son del orden de $5 \times 10^{-2}$ milímetros. 


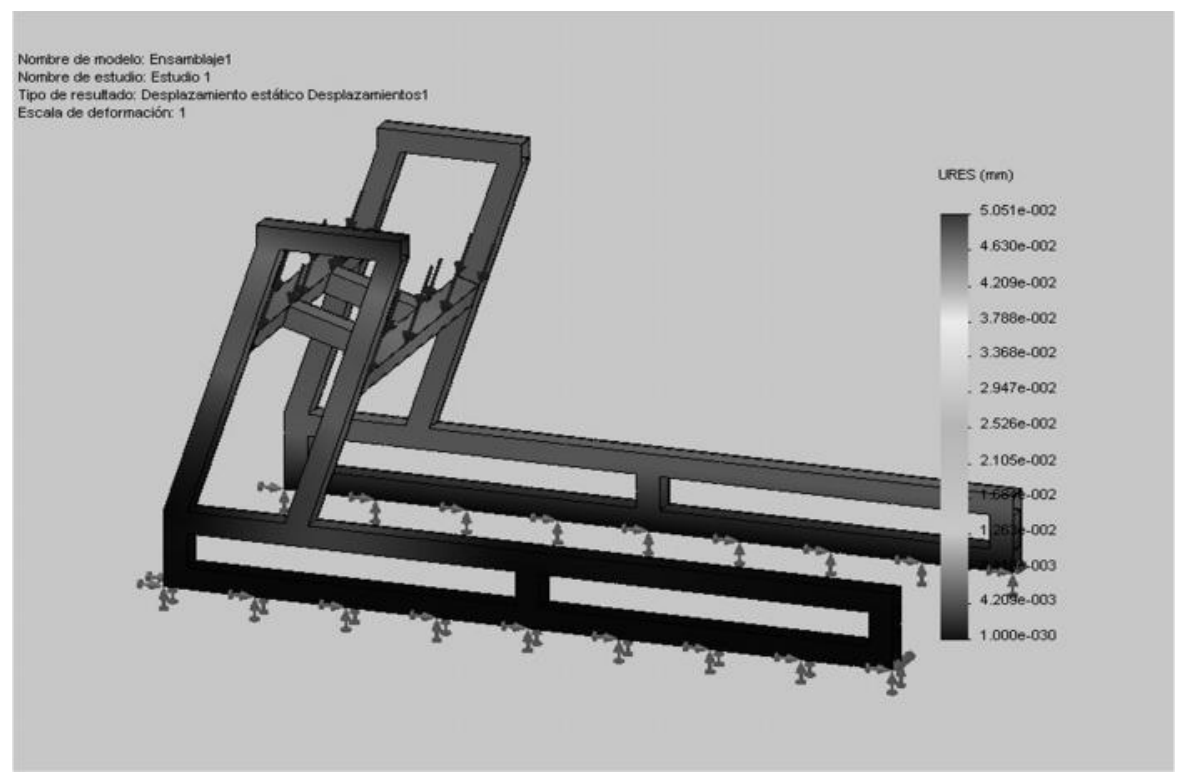

Figura 10. Deformaciones Unitarias - SolidWorks ${ }^{\circledR}$

La figura 11 indica los esfuerzos de Von Mises que se pueden llegar a presentar en el cuerpo de la estructura, en este caso el máximo esfuerzo que se presenta es de $3.35 \mathrm{Mpa}$ el cual es bastante bajo si se compara con el esfuerzo de fluencia del material Acero Inoxidable 303, Sy = $241 \mathrm{Mpa}$ (5).

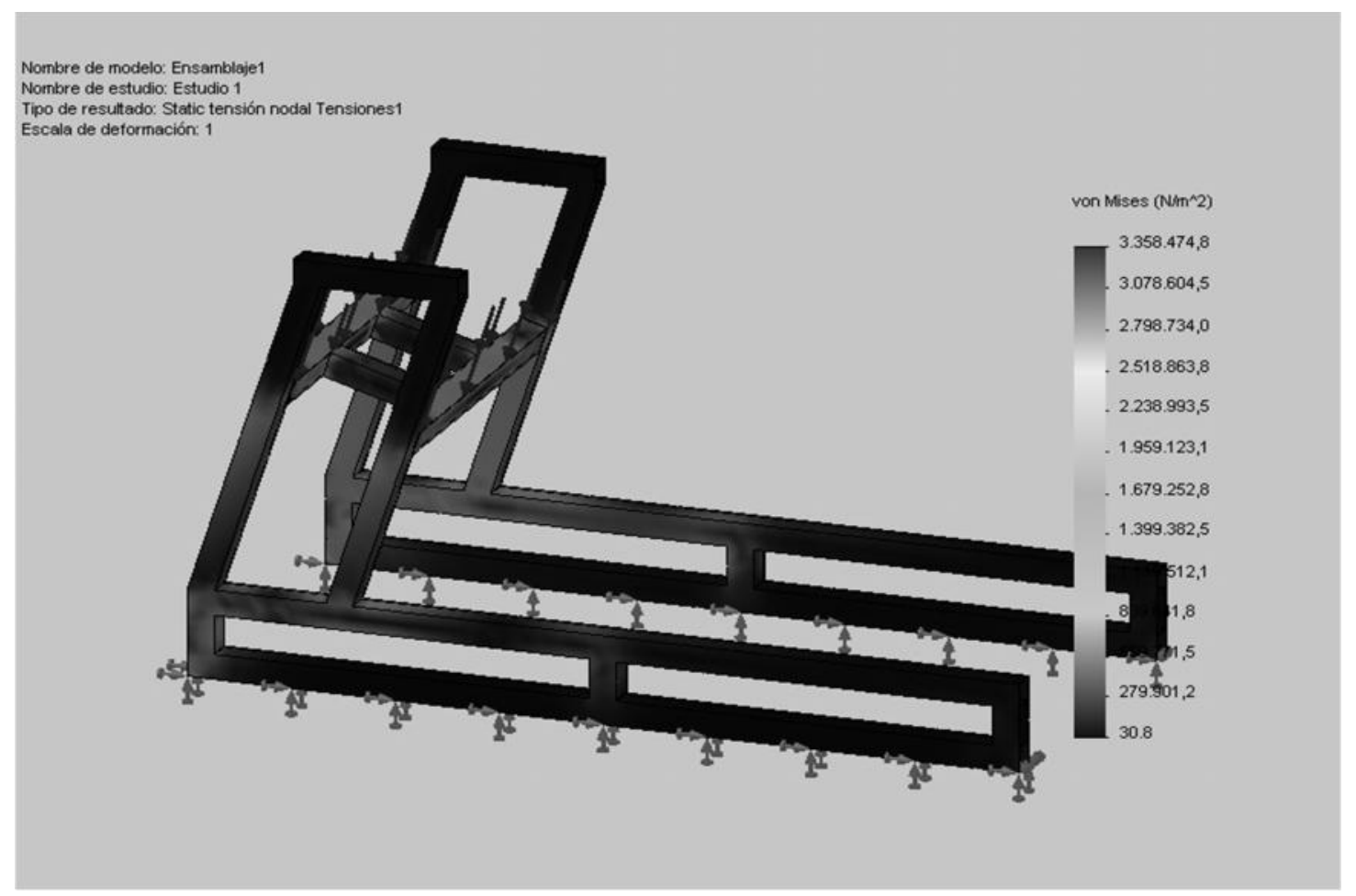

Figura 11. Esfuerzos de Von Mises - SolidWorks ${ }^{\circledR}$ 
La figura 12 indica los factores de seguridad presente en la estructura, en este caso el mínimo factor de seguridad es de 230, lo cual indica que la estructura es bastante segura.

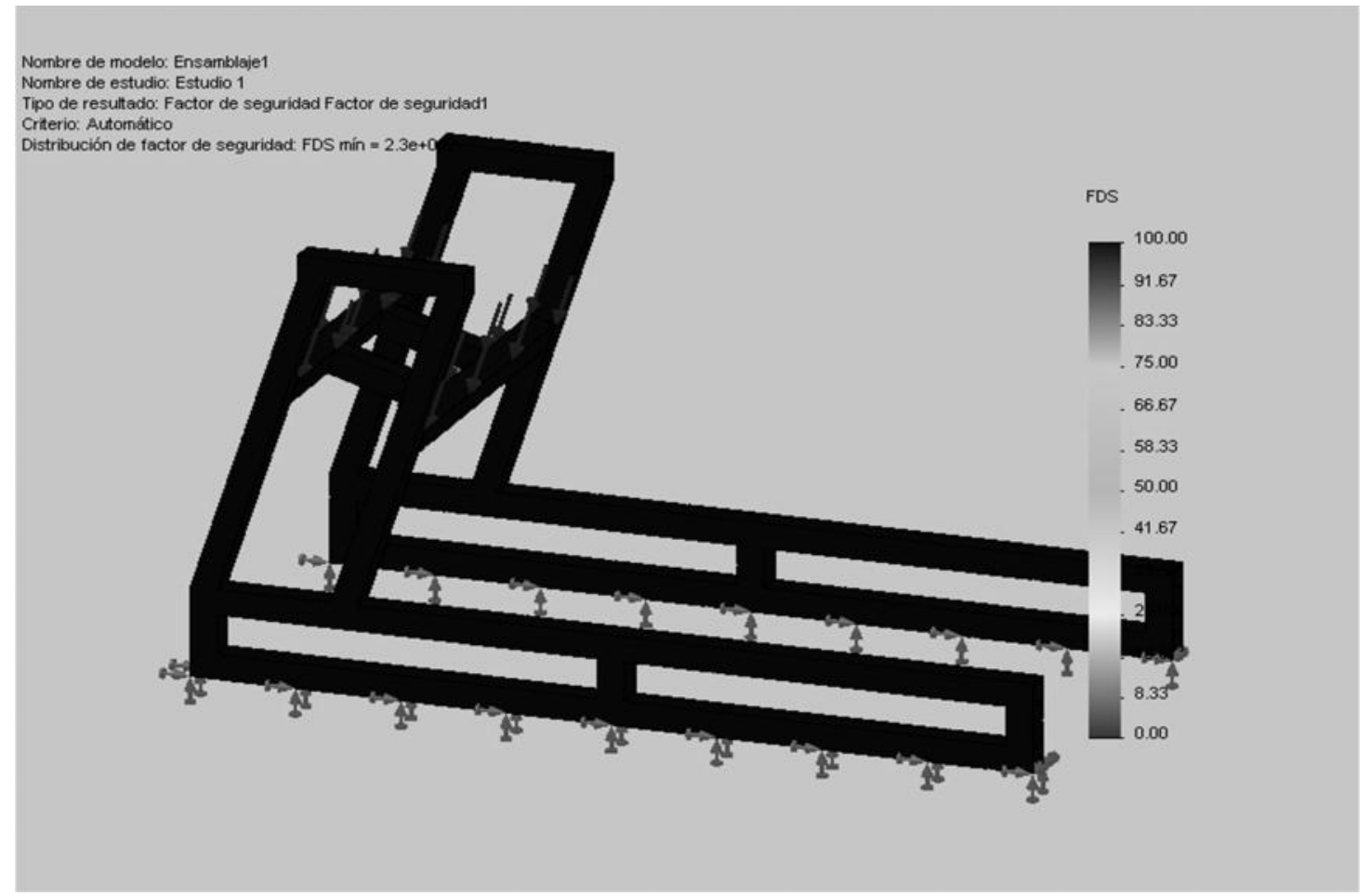

Figura 12. Factores de Seguridad - SolidWorks ${ }^{\circledR}$

Camilla: La estructura interna esta hecha en tubo cuadrado estructural de acero inoxidable (2"x 2"x $1 / 8$ ") y recubierta con lámina del mismo material, en el estudio de diseño y simulación se tomo únicamente la parte que soporta mayor carga, por consiguiente y al igual que con las patas se tomo una carga de simulación y diseño de $750 \mathrm{~N}$.

En las figuras 13 - 16 se puede observar el proceso de diseño y simulación de las patas, la figura 14 muestra los puntos de aplicación de apoyos y cargas.

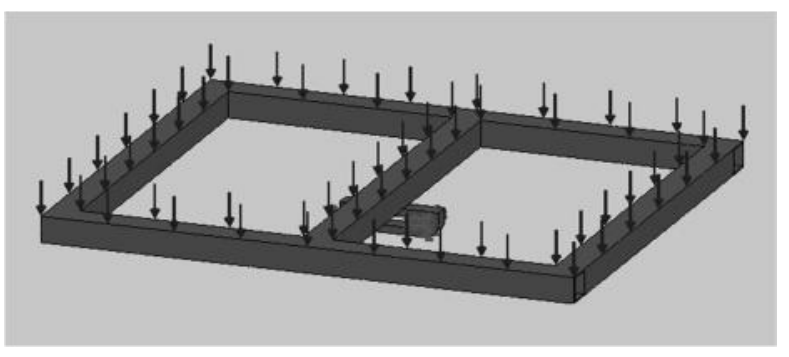

Figura 13. Cargas Estructura de la Parte Superior de la Camilla - SolidWorks ${ }^{\circledR}$
La figura 14 indica las deformaciones máximas que se pueden llegar a presentar en la estructura, en este caso son del orden de 0.17 milímetros.

La figura 15 indica los esfuerzos de Von Mises que se pueden llegar a presentar en el cuerpo de la estructura, en este caso el máximo esfuerzo que se presenta es de 10.4 Mpa el cual es bastante bajo si se compara con el esfuerzo de fluencia del material Acero Inoxidable 303, Sy = $241 \mathrm{Mpa}$ (5).

La figura 16 indica los factores de seguridad presente en la estructura, en este caso el mínimo factor de seguridad es de 75 , esto indica que la estructura es bastante segura.

Tornillo Sinfín: según los cálculos matemáticos realizados se estima que el tornillo sinfín este construido de acero AISI 1020 CD con un diámetro exterior de 1.5 in, bajo la acción de una carga de 750 $\mathrm{N}$ se realizaron los siguientes estudios. 


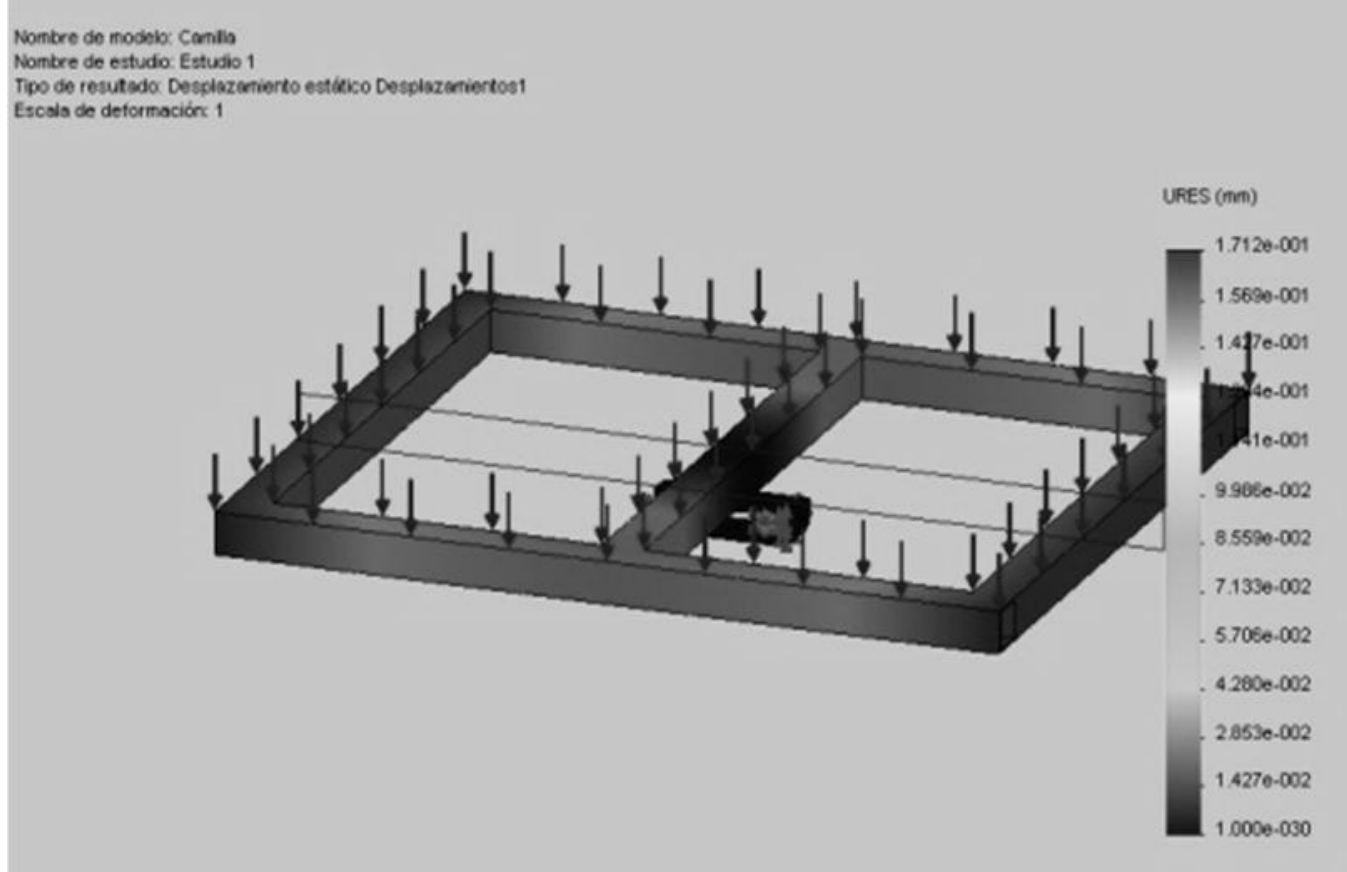

Figura 14. Deformaciones Unitarias - SolidWorks ${ }^{\circledR}$

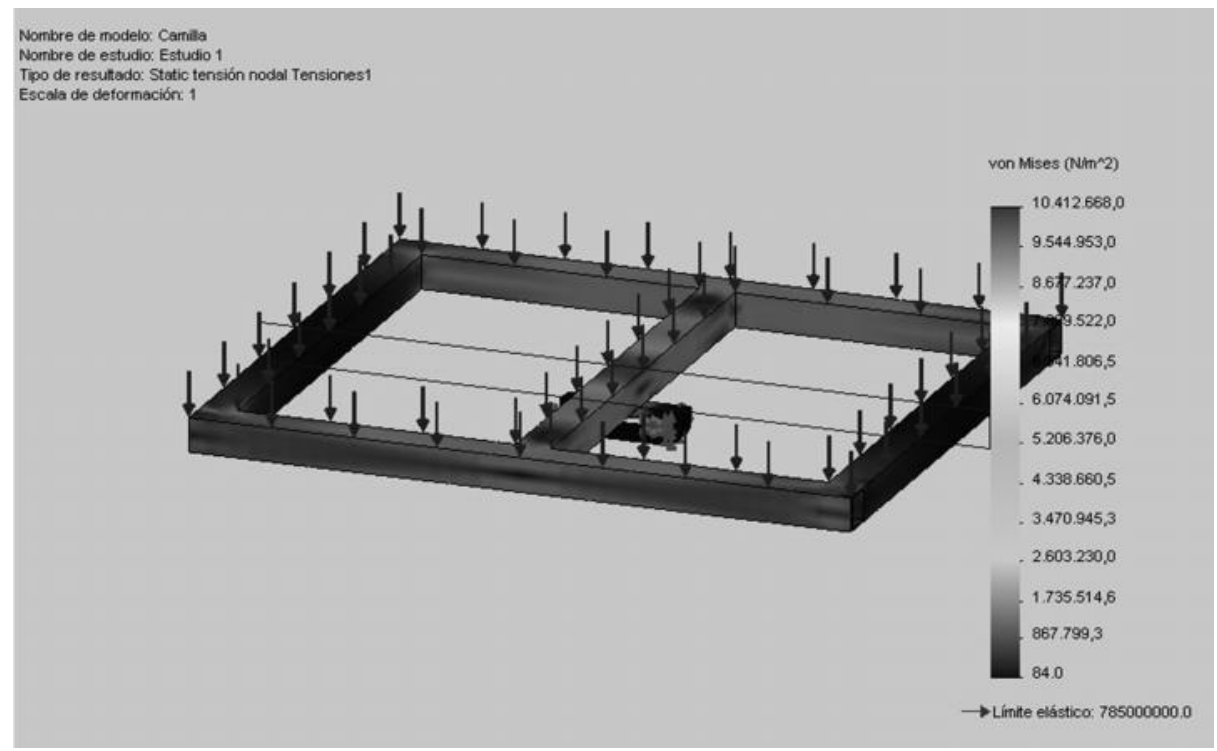

Figura 15. Esfuerzo de Von Mises - SolidWorks ${ }^{\circledR}$ 


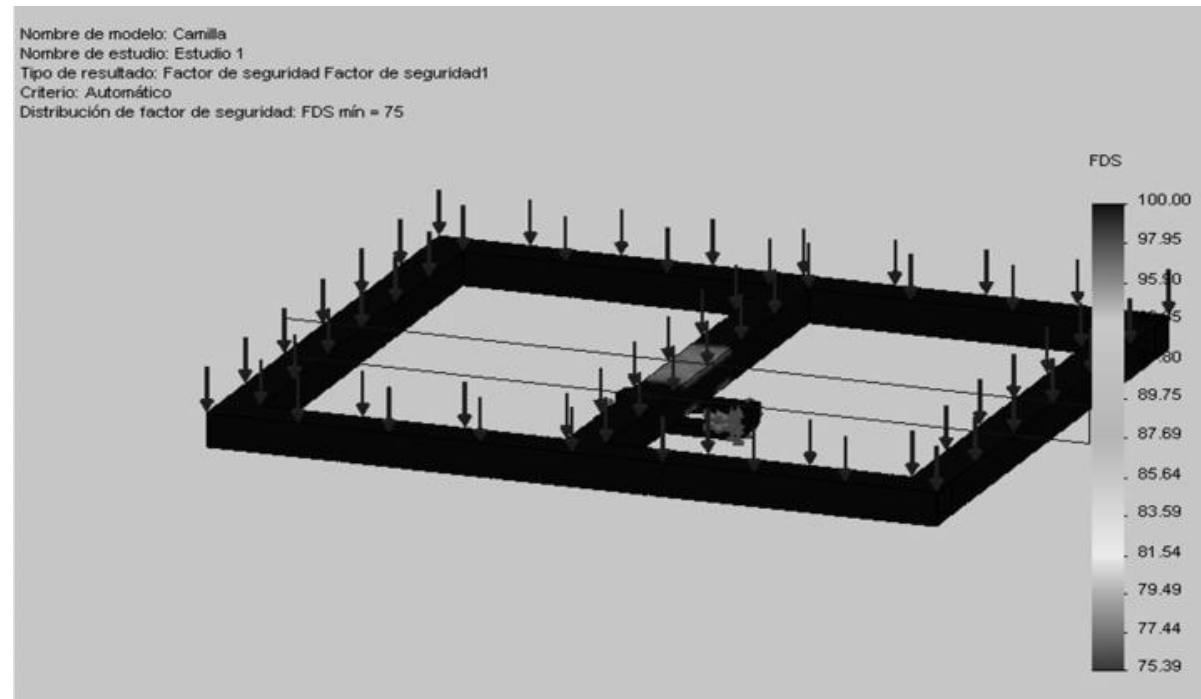

Figura 16. Deformaciones Unitarias - SolidWorks ${ }^{\circledR}$

La figura 17 indica las deformaciones unitarias que se pueden llegar a presentar en el tornillo, en este caso son del orden de 2.4 x 10-4 milímetros.

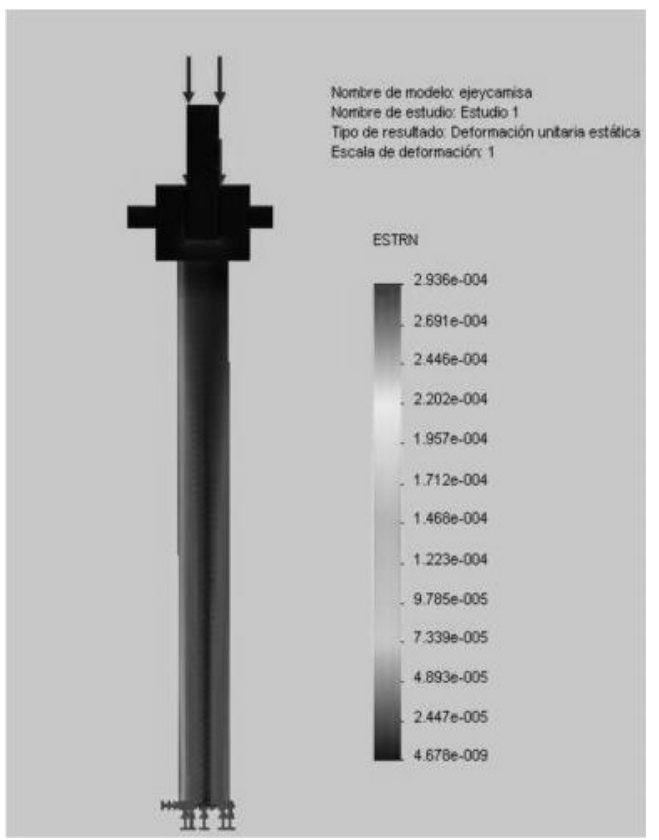

Figura 17. Deformaciones Unitarias - SolidWorks ${ }^{\circledR}$

La figura 19 indica los esfuerzos de Von Mises que se pueden llegar a presentar en el cuerpo del torni1lo, en este caso son del orden de $50 \mathrm{Mpa}$, lo que es bastante bajo si se tiene en cuenta que el esfuerzo de fluencia del material es de $390 \mathrm{Mpa}$ (6), esto indica que el factor de seguridad de esta pieza es de aproximadamente 7.8 .

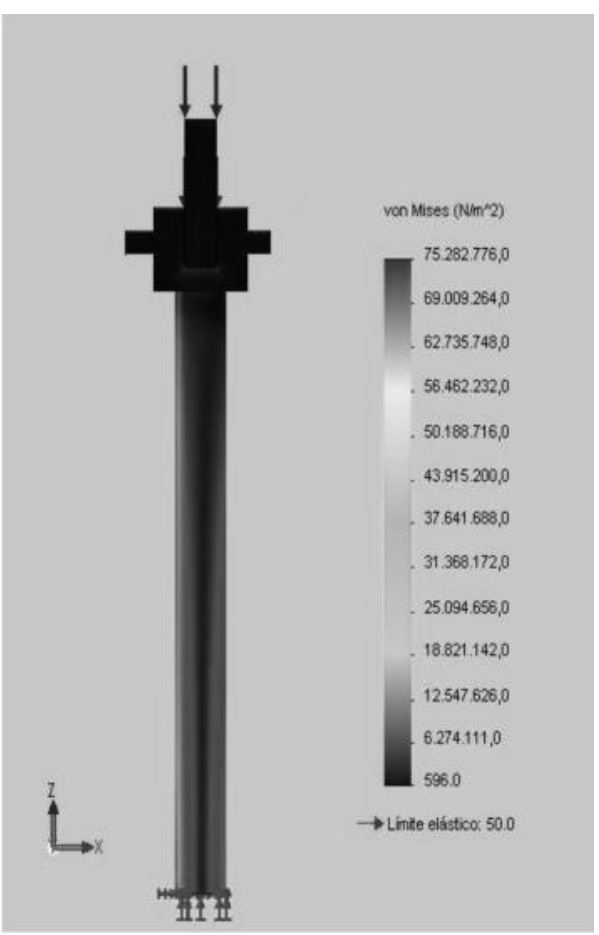

Figura 18. Esfuerzo de Von Mises - SolidWorks ${ }^{\circledR}$

Adicionalmente en la figura 19 se puede observar el estudio de pandeo del tornillo bajo la acción de la carga estimada, este estudio indica que la estructura no se pandea y que puede llegar a tener una desviación de 1 milímetro. 


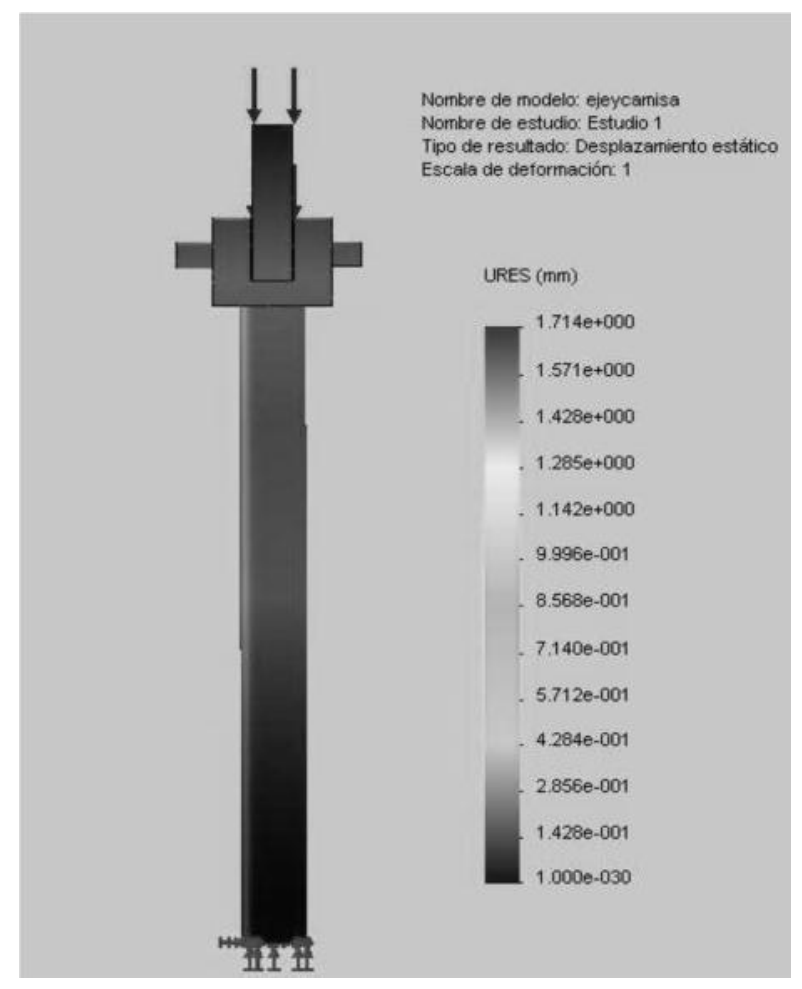

Figura 19. Estudio de Pandeo - SolidWorks ${ }^{\circledR}$

\section{Control}

Para el sistema de control del motor que activa el tornillo sinfín tanto para subir como para bajar la camilla se dispuso de un PLC LOGO! 24 RCL Marca Siemens, como el que se indica en la figura 21 , este tipo de control ha sido utilizado con gran eficiencia en los equipos desarrollados por el centro de Fisioterapia Cyriax.

Este control se debe programar según los requerimientos del equipo, adicionalmente se deben colocar sensores de distancia y fin de carrera, lo cual permitirá brindar mayor seguridad tanto a pacientes, operarios y al equipo como tal.

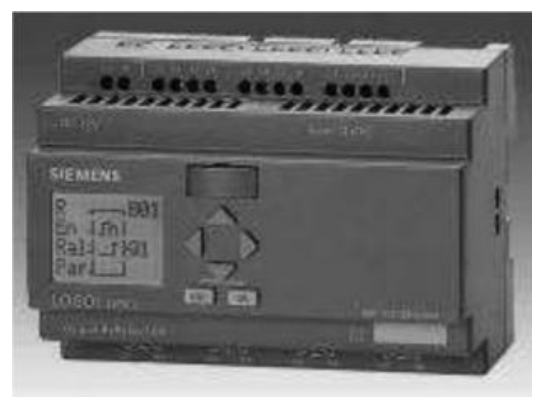

Figura 20. PLC LOGO! 24 CRL

\section{DISCUSIÓN}

El equipo propuesto le permitirá al Centro de Fisioterapia Cyrias el tratamiento de varias molestias de la columna vertebral, las cuales de otra forma necesitarían de la adquisición de varios equipos.

La integración de varias funciones en un solo equipo permitirá a futuro la reducción del espacio en el cual se realizan estos procedimientos fisioterapéuticos, ya que solo se instalara un solo equipo.

Estos factores mencionados anteriormente requerirán de una menor inversión por parte del Centro de Fisioterapia Ciryas y permitirá ofrecer un servicio de mayor calidad.

\section{CONCLUSIONES}

A partir del proceso de investigación realizado se logró dimensionar, diseñar y modelar un equipo multifuncional que pueda ayudar en el tratamiento y rehabilitación en pacientes con trauma raquimedular de nivel bajo, lesión en cauda equina y que además sirva para tracción lumbar en pacientes con hernia discal a nivel lumbar.

Con este diseño el Centro de Fisioterapia Cyriax pretende adquirir más tecnología que pueda brindarle al paciente neurológico y osteomuscular mayores herramientas en sus tratamientos, y de esta manera lograr una mayor calidad en los servicios.

El conocimiento de las características que debe tener un equipo de mecanoterapia para maximizar su funcionalidad, permitió identificar pautas a cumplir y estableció requerimientos y especificaciones que al final fueron implementadas en el equipo diseñado.

Con la construcción de este equipo el Centro de Fisioterapia Cyriax espera en pocos años convertirse en el mejor centro de rehabilitación del sur de Colombia brindando tratamientos de calidad a sus pacientes. 


\section{RECOMENDACIONES}

Seguir los estándares de la norma IEC 60601 con especificidad en el estándar IEC 60601-2-10 para equipos de mecanoterapia que también incluyan electro estimulación, para desarrollar el prototipo del equipo.

Limitar el uso del equipo a personal capacitado en el manejo de estas herramientas, puesto que una manipulación incorrecta de este dispositivo puede acarrear daños irreversibles en los pacientes y en el equipo como tal.

Realizar más pruebas de funcionamiento que permitan en un futuro próximo la construcción e implementación de este equipo por parte del Centro de Fisioterapia - Cyriax y su comercialización en Colombia y Ecuador.

\section{AGRADECIMIENTOS}

Los autores expresan sus agradecimientos a la Universidad de Pamplona y al Centro de Fisioterapia Cyriax - Pasto - Colombia por todo el apoyo dado durante el desarrollo de este proyecto.

\section{BIBLIOGRAFÍA}

1. Arthur C. Guyton y John E. Hall. Fisiología Médica. Editorial McGraw-Hill. Decima Edición. 2000. Página 751.

2. Gatch Beb and Adjustable Hospital Beds (Agosto -2011). [Online]. Disponible: http://www.askdeb.com/health/ bed/

3. Ciaffaroni Castro D., Cucco A., Dellatorre L., Mazzone J. y Bazán N. Estimación del Peso Total en Personas Amputadas en Miembros Inferiores. Universidad Nacional de San Martín. ReCAD - Revista electrónica de Ciencias Aplicadas al Deporte, 2011; 4(13).

4. David J. Oborne. Ergonomía en acción. La adaptación del medio de trabajo al hombre. Editorial Trillas. México 1987.

5. R. Budynas; Diseño en Ingeniería Mecánica de Shigley, 8va edición, McGraw-Hill, 2008

6. R. Mott; Diseño de Elementos de Maquinas, Cuarta Edición, Pearson - Prentice Hall, 2006 